\title{
Remote macroscopic entanglement on a photonic crystal architecture
}

\author{
H. Flayac, ${ }^{*}$ M. Minkov, and V. Savona \\ Institute of Theoretical Physics, École Polytechnique Fédérale de Lausanne EPFL, CH-1015 Lausanne, Switzerland
}

(Received 20 May 2015; published 12 October 2015)

\begin{abstract}
The outstanding progress in nanostructure fabrication and cooling technologies allows what was unthinkable a few decades ago: bringing single-mode mechanical vibrations to the quantum regime. The coupling between photon and phonon excitations is a natural source of nonclassical states of light and mechanical vibrations, and its study within the field of cavity optomechanics is developing lightning fast. Photonic crystal cavities are highly integrable architectures that have demonstrated the strongest optomechanical coupling to date and should therefore play a central role for such hybrid quantum-state engineering. In this context, we propose a realistic heralding protocol for the on-chip preparation of remotely entangled mechanical states, relying on the state-of-the-art optomechanical parameters of a silicon-based nanobeam structure. Pulsed sideband excitation of a Stokes process, combined with single-photon detection, allows the writing of a delocalized mechanical Bell state in the system, signatures of which can then be read out in the optical field. A measure of entanglement in this protocol is provided by the visibility of a characteristic quantum interference pattern in the emitted light.
\end{abstract}

DOI: 10.1103/PhysRevA.92.043812 PACS number(s): 03.67.Bg, 42.50.Wk, 42.50.Dv, 42.70.Qs

\section{INTRODUCTION}

Cavity optomechanics [1-4] remarkably allows the transposition of cavity quantum electrodynamics features to vibrational quanta, through radiation pressure. This field of research has demonstrated a spectacular theoretical and experimental development in the past decade and is now envisioned as one of the most promising routes to the production of nonclassical states of macroscopic degrees of freedom. Recently, a number of theoretical investigations have predicted such nonclassical mechanical states to occur in the form of squeezed states [5-8], Fock states $[9,10]$, and photon-phonon [11-15] or purely mechanical entangled states [16-26]. Such achievements would not only shine light on the foundations of quantum mechanics and the decoherence process, but could also provide a longlived information storage or processing platform useful for potential quantum repeaters [27]. Outstanding experimental results have already been obtained in this direction by Lee and coworkers [28] who managed, based on the Duan-LukinCirac-Zoller protocol [29], to entangle two optical-phonon modes stored in remote diamond crystals at room temperature. Meanwhile, current cooling technology [30-33] is paving the way to nonclassical states stored in acoustic-phonon modes, which can be pictured as the collective motion of a macroscopic number of atoms in the solid.

The recent progress in the optimization of silicon [31,34], indium phosphide $[35,36]$, or more recently diamondbased [37,38] photonic crystal structures allow the combination of high quality factors of cavity modes with confined mechanical modes, resulting in record values of the optomechanical coupling reaching the $\mathrm{MHz}$ range [31]. Thanks to their small footprint, solid-state structures lie among the best candidates for the future of integrated quantum logic elements that could exploit optical and mechanical quanta to code information. In this framework, it is desirable to have access to on-chip operations that involve single quanta of excitation and remotely entangled states [39] of a high purity.

\footnotetext{
*hugo.flayac@epfl.ch
}

In this article we predict the strong potential of optimized silicon nanobeam structures for on-chip entanglement of distant mechanical vibrations. We first design and characterize a promising candidate system based on a state-of-the-art photonic-phononic crystal structure. Then, we demonstrate the possibility to engineer remotely entangled states of mechanical quanta on-chip, based on a high efficiency heralding protocol [26]. The quantum dynamics is described by means of a master equation treatment that includes the impact of environmental interactions and finite temperature.

\section{THE STRUCTURE}

We propose the system shown in Fig. 1, which is based on a pair of silicon nanobeams of width $529 \mathrm{~nm}$ and thickness $220 \mathrm{~nm}$, at a distance of $140 \mathrm{~nm}$ from each other. The first beam is patterned in order to host two defect cavities based on the optimized design proposed in Refs. [31,40], separated by a distance of $d=6.3 \mu \mathrm{m}$. The lattice constant of the photonic crystal pattern (cf. Ref. [40] for details) is $a_{0}=436 \mathrm{~nm}$. The second beam hosts a clogged waveguide section that supports Fabry-Perot-like modes and serves to couple the two cavities in an implementation similar to the one of Ref. [41]. Such a structure could be used to remotely couple cavities that are separated by a distance of hundreds of wavelengths.

To demonstrate waveguide-mediated optical coupling we model the structure by using a commercial-grade simulator based on the finite-difference time-domain (FDTD) method [42]. By imposing odd, even, or no parity with respect to the $x=0$ transverse plane, we can selectively excite the antisymmetric [Fig. 2(a)], symmetric [Fig. 2(b)], or both modes of the coupled system. The corresponding simulated spectra are presented in Fig. 2(c). The modes resonate around the frequency $\omega_{c}=2 \pi \times 193.45 \mathrm{THz}$. To accurately determine the frequency splitting, we record the electric field in the center of one cavity when both modes are excited and observe a clear beating pattern, as shown in the inset. From the time interval between the two antinodes we compute $2 J_{c}=2 \pi \times 16.9 \mathrm{GHz}$, which is consistent with the spectrum in Fig. 2(c). In the absence of the waveguide instead, 


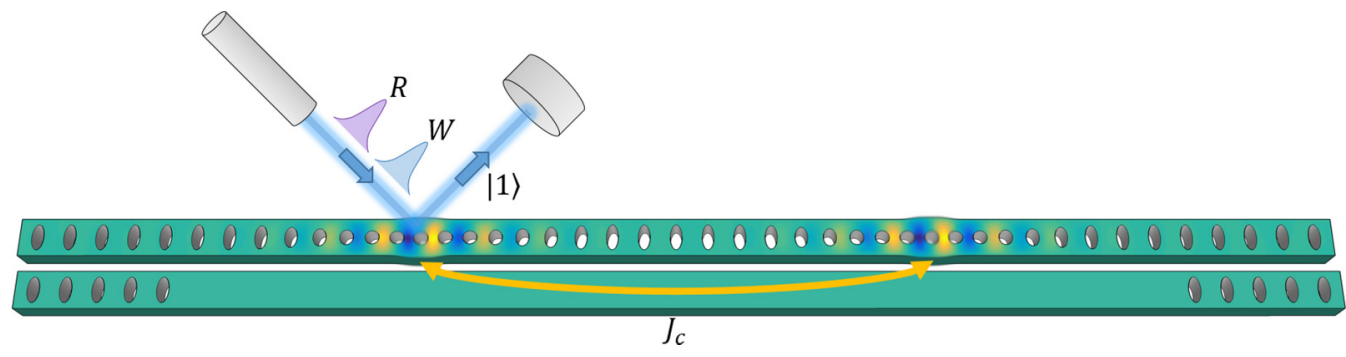

FIG. 1. (Color online) Three-dimensional model of the system under study, showing the nanobeam photonic crystal (upper) and the clogged waveguide (lower), together with a sketch of the excitation and detection conditions. Cavity 1 is driven under pulsed excitation and its output is collected by a single-photon detector. The yellow arrow highlights the waveguide-induced optical coupling of strength $J_{c}$ between the two cavities. The deformation of the cavities illustrate the slight transverse vibrations induced by the optical field that are computed in Figs. 2(d) and 2(e).

the simulation results in a splitting of about $2 \pi \times 4 \mathrm{GHz}$ that would dramatically drop for larger cavity separation, thus highlighting the role of the waveguide in enhancing the coupling between the two cavities. The quality factors of the two modes were computed from the time decay of the field after selective excitation and are $Q_{-}=1.14 \times 10^{6}$ and $Q_{+}=8.0 \times 10^{5}$, corresponding to linewidths of $\kappa_{-}=2 \pi \times$ $169 \mathrm{MHz}$ and $\kappa_{+}=2 \pi \times 243 \mathrm{MHz}$ for the antisymmetric and symmetric modes, respectively. The linewidths are slightly unbalanced due to the different overlap of the two confined modes with the continuum of radiation modes outside the
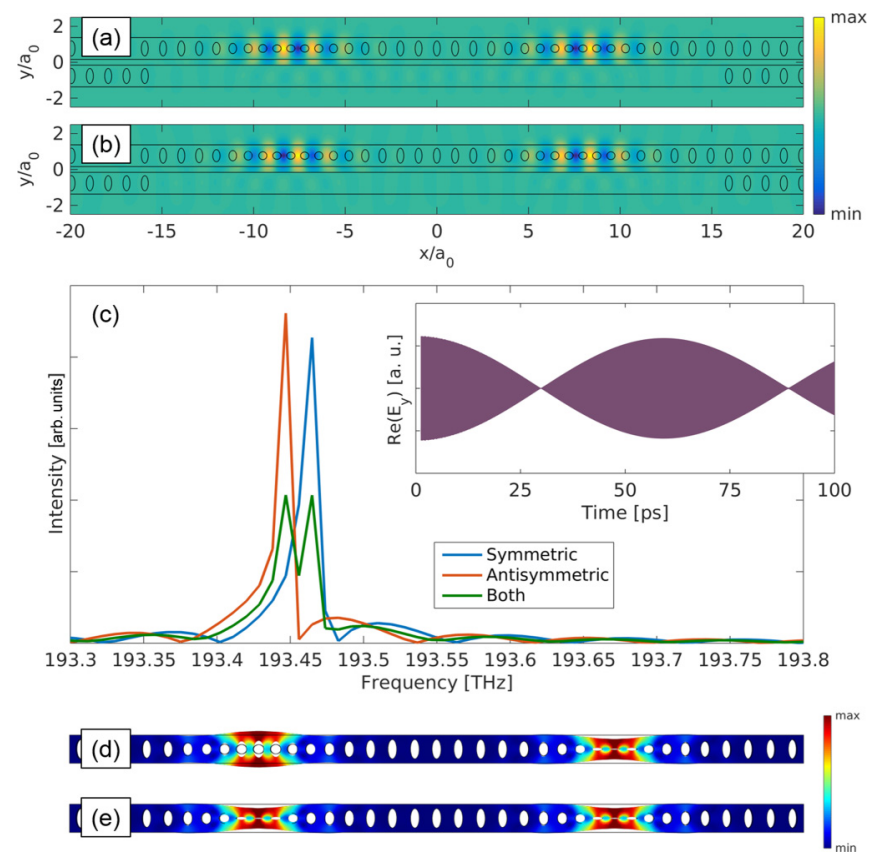

FIG. 2. (Color online) (a) Antisymmetric and (b) symmetric normal modes of the electromagnetic field in the coupled cavity system. The color map expresses the $\operatorname{Re}\left(E_{y}\right)$ component of the electric field. (c) Simulated optical spectrum after individual or simultaneous excitation of the optical modes. The inset shows the time dependence of the electric field, as recorded at the center of one cavity when both modes are excited. (d) Antisymmetric and (e) symmetric confined mechanical modes. The color map expresses the amplitude of the displacement field. beams. Even though such high $Q$ values are experimentally achievable [40], below we conservatively assume twice larger values of $\kappa_{-}=2 \pi \times 338 \mathrm{MHz}$ and $\kappa_{+}=2 \pi \times 486 \mathrm{MHz}$ in view of the extra losses that would be introduced by the measurement though input and output channels such as tapered fibers [43].

The vibrational eigenmodes of the structure, associated with the two defect cavities, were computed via finite-element modeling [44]. The localized transverse breathing modes, as described in Ref. [31], are found at a frequency $\Omega_{m}=2 \pi \times$ $5.08 \mathrm{GHz}$. As for the optical modes, symmetric and antisymmetric normal modes of the vibrations at the two cavities arise, which are characterized by a negligible frequency separation of $2 J_{m}=2 \pi \times 8.8 \mathrm{kHz}$. The displacement fields of these modes are depicted in Figs. 2(d) and 2(e). The associated distortion of the cavity is transverse to the main axis of the structure, producing a remarkably high single-photon optomechanical coupling of $g_{0}=2 \pi \times 0.86 \mathrm{MHz}$ [40]. We assume the mechanical quality factor $Q_{m}=6.8 \times 10^{5}$ measured in Ref. [31], where the structure was coupled to an acoustic radiation shield. As we will see, the readout of the mechanical entangled state requires that the vibrational modes are nondegenerate, in order to introduce a relative phase factor between the two optomechanical coupling processes, which reveals the quantum superposition of the two mechanical modes through an interference pattern in the optical readout [26]. Different frequencies for the mechanical modes of the two cavities will naturally arise due to imperfections in the nanofabrication process. We have therefore studied the impact of the structural disorder on the resonant mechanical frequency of one cavity by introducing random, Gauss-distributed fluctuations of the hole dimensions and positions with a standard deviation of $1 \mathrm{~nm}$. Over 100 simulated disorder realizations we found a typical $1 \%$ fluctuation of the mechanical frequency. In the quantum model that follows, we will therefore assume $\Omega_{1}=\Omega_{m}$ and $\Omega_{2}=1.01 \times \Omega_{1}=2 \pi \times 5.13 \mathrm{GHz}$.

\section{QUANTUM MODEL}

The system can be schematically summarized as in Fig. 3(a); namely, as two coupled, single-mode cavities ( $\hat{a}_{1,2}$ operators), each optomechanically coupled to distinct mechanical modes $\left(\hat{b}_{1,2}\right.$ operators). The system Hamiltonian 

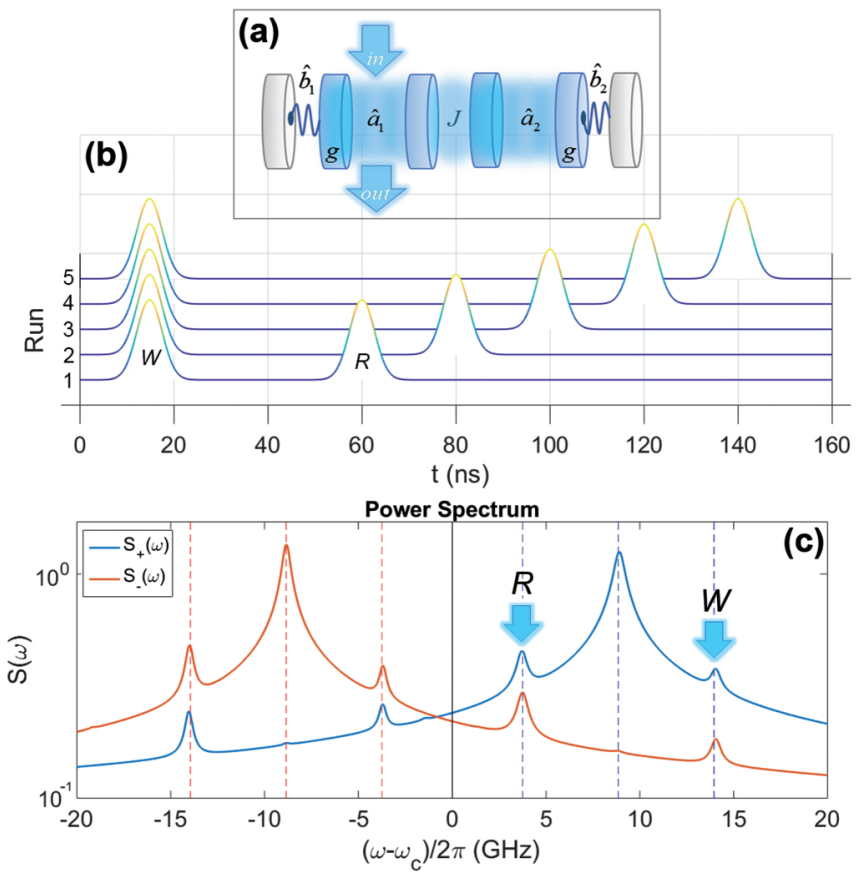

FIG. 3. (Color online) (a) Schematic representation of the model system. (b) Write and readout pulses sequences for the interference reconstruction. (c) The computed power spectrum in log scale of the symmetric and antisymmetric cavity modes, revealing the Stokes and anti-Stokes sidebands.

reads

$$
\begin{aligned}
\hat{\mathcal{H}}= & \sum_{j=1,2}\left[\omega_{c} \hat{a}_{j}^{\dagger} \hat{a}_{j}+\Omega_{j} \hat{b}_{j}^{\dagger} \hat{b}_{j}+g_{0} \hat{a}_{j}^{\dagger} \hat{a}_{j}\left(\hat{b}_{j}^{\dagger}+\hat{b}_{j}\right)\right] \\
& +J_{c}\left[\hat{a}_{1}^{\dagger} \hat{a}_{2}+\hat{a}_{2}^{\dagger} \hat{a}_{1}\right]+F(t)\left[\hat{a}_{1}^{\dagger} e^{-i \omega_{L} t}+\hat{a}_{1} e^{+i \omega_{L} t}\right] .
\end{aligned}
$$

We introduce an optical write and readout protocol analogous to the one we recently adopted in the case of a single optical cavity [26]. The system is driven first by a classical source of amplitude $F(t)$ and frequency $\omega_{L}$ in cavity 1 that stands for our input-output mode [see $W$ in Fig. 3(c)]. It is instructive to rewrite $\hat{\mathcal{H}}$ on the basis of the symmetric and antisymmetric modes following $\hat{a}_{ \pm}=\left(\hat{a}_{1} \pm \hat{a}_{2}\right) / \sqrt{2}$ and $\hat{b}_{ \pm}=$ $\left(\hat{b}_{1} \pm \hat{b}_{2}\right) / \sqrt{2}$ which recasts the optomechanical contribution $\hat{\mathcal{H}}_{g}=g_{0} \sum_{j} \hat{a}_{j}^{\dagger} \hat{a}_{j}\left(\hat{b}_{j}^{\dagger}+\hat{b}_{j}\right)$ as

$$
\begin{aligned}
\hat{\mathcal{H}}_{g}= & \frac{g_{0}}{\sqrt{2}}\left(\hat{a}_{+}^{\dagger} \hat{a}_{+}+\hat{a}_{-}^{\dagger} \hat{a}_{-}\right)\left(\hat{b}_{+}^{\dagger}+\hat{b}_{+}\right) \\
& +\frac{g_{0}}{\sqrt{2}}\left(\hat{a}_{+}^{\dagger} \hat{a}_{-}+\hat{a}_{-}^{\dagger} \hat{a}_{+}\right)\left(\hat{b}_{-}^{\dagger}+\hat{b}_{-}\right) .
\end{aligned}
$$

It describes two cavity modes of resonance $\omega_{ \pm}=\omega_{c} \pm J_{c}$ (i) optomechanically coupled to the same vibrational mode $\hat{b}_{+}$of frequency $\Omega_{+}=\left(\Omega_{1}+\Omega_{2}\right) / 2 \simeq \Omega_{1,2}$ (first term) and (ii) coupled together through the absorption or emission of a phonon of the mode $\hat{b}_{-}$(second term). The latter process, which would allow an exchange of quanta between the $\hat{a}_{+}$ and $\hat{a}_{-}$modes, is off resonant in the conditions we consider where $\omega_{+}-\omega_{-} \simeq 3 \Omega_{1,2}$ and can be discarded. The cavity spectrum shown in Fig. 3(c) consists of two main resonances at $\omega_{c} \pm J$ each surrounded by sidebands formed by the Raman interaction with the $\hat{b}_{+}$mode (dashed lines). Note that (ii) makes the $\hat{a}_{+}$sidebands visible in the $\hat{a}_{-}$spectrum and vice versa.

If cavity 1 is driven on the resolved $\hat{a}_{+}$Stokes sideband, i.e., setting $\omega_{L}=\omega_{c}+J_{c}+\Omega_{+}$[see $W$ arrow in Fig. 3(c)], the first term of Eq. (2) then produces the two-mode squeezing [4]

$$
\hat{\mathcal{H}}_{g} \approx \frac{g}{\sqrt{2}}\left(\delta \hat{a}_{+}^{\dagger} \hat{b}_{+}^{\dagger}+\delta \hat{a}_{+} \hat{b}_{+}\right)
$$

between the mechanical mode and the cavity fluctuations $\delta \hat{a}_{+}$ under the standard linearized picture [4] (which we detail below). Here $\hat{a}_{+}=\left\langle\hat{a}_{+}\right\rangle+\delta \hat{a}_{+}$and $g=\left\langle\hat{a}_{+}\right\rangle g_{0}$ is the enhanced optomechanical coupling. The $\hat{a}_{-}$contribution is negligible since its frequency is shifted by $-\left(2 J_{c}+\Omega_{+}\right)$from the pump frequency, thus resulting in a strongly nonresonant process. The interaction (3) therefore creates a pair correlation between the symmetric cavity fluctuations and mechanical modes. Under continuous-wave driving, one could therefore achieve a steady-state hybrid continuous variable entanglement between the $\hat{a}_{+}$and $\hat{b}_{+}$modes [12]. However, our goal here is to produce a mechanical excitation in a linear superposition of two Fock states, each corresponding to one quantum of vibration in one cavity. This requires combining pulsed excitation with postselection. Indeed, after the write pulse, the detection of a single photon emitted by any of the two cavities heralds the presence of a single phonon [10,26] stored in the mechanical mode $\hat{b}_{+}$, i.e., shared between the remote modes $\hat{b}_{1}$ and $\hat{b}_{2}$.

In order to gain insight into the entanglement generation protocol that we propose, before resorting to the numerical solution of the equations for the driven-dissipative system we provide here a simplified description of the elementary steps of the protocol in terms of pure states. For weak pulses of short duration $\Delta t$, we can roughly approximate the time evolution operator as $\hat{\mathcal{U}} \sim \hat{\mathbb{I}}-i \hat{\mathcal{H}} \Delta t+O\left(\Delta t^{2}\right)$ and consider the effect of the optomechanical Hamiltonian on the initial state. Assuming zero temperature, the system lies initially in its vacuum state $\left|\psi_{0}\right\rangle=|0\rangle_{c_{1}} \otimes|00\rangle_{m} \otimes|0\rangle_{c_{2}}$, where the indices $c_{j}$ and $m$ refer to the cavities and mechanical states, respectively. Then, the write laser tuned to the Stokes process of the $\hat{a}_{+}$mode drives the Hamiltonian term (3) and ideally results in the quantum state

$$
\begin{aligned}
\left|\psi_{1}\right\rangle \simeq & \hat{\mathcal{U}}\left|\psi_{0}\right\rangle \\
= & c_{00}|0\rangle_{c_{1}} \otimes|00\rangle_{m} \otimes|0\rangle_{c_{2}} \\
& +c_{10}|1\rangle_{c_{1}} \otimes\left(|10\rangle_{m}+e^{i \varphi}|01\rangle_{m}\right) \otimes|0\rangle_{c_{2}} \\
& +c_{01}|0\rangle_{c_{1}} \otimes\left(|10\rangle_{m}+e^{i \varphi}|01\rangle_{m}\right) \otimes|1\rangle_{c_{2}}+\cdots
\end{aligned}
$$

Here, $\varphi=\varphi_{0}+\left(\Omega_{2}-\Omega_{1}\right) t$ where $\varphi_{0}$ is a tunable phase shift depending on the details of the excitation scheme. Note that an unequal optomechanical coupling to the two mechanical modes would induce an additional phase shift to $\varphi$. The coefficients $c_{i j}$ are determined by the details of the time evolution. In particular, one expects that, in the limit of weak field amplitude, the components of this state corresponding to two- and more photons are negligible. A single-photon detection from cavity 1 retains only the second term of Eq. (4) and the state of the system, after detection, reduces to

$$
\left|\psi_{W}\right\rangle=c_{10}^{\prime}|0\rangle_{c_{1}} \otimes\left(|10\rangle_{m}+e^{i \varphi^{\prime}}|01\rangle_{m}\right) \otimes|0\rangle_{c_{2}},
$$


which is nothing but a maximally entangled mechanical Bell state $\left|\psi_{\mathcal{H}}\right\rangle_{m}=\left(|10\rangle_{m}+\exp \left(i \varphi^{\prime}\right)|01\rangle_{m}\right) / \sqrt{2}$ when the cavity modes are traced out. According to this hand-waving picture of the protocol, the system is cast in state (5) upon heralding, i.e., whenever one photon is detected from cavity 1 . The probability amplitude of this event is associated with the ratio $c_{10} / c_{00}$, which in turn is roughly proportional to the enhanced optomechanical coupling $g$. Thus, a finite heralding probability in principle exists for arbitrarily small values of $g$. In a realistic setup, however, we expect that several effects will contribute to set a lower bound on the optomechanical coupling required to successfully herald this process, among which are singlephoton-detection efficiency and noise from various origins. Below, we carry out full numerical simulations of the protocol and select the pulse amplitudes so to obtain a significant heralding rate when assuming a single-photon optomechanical coupling $g_{0}=2 \pi \times 0.86 \mathrm{MHz}$ [40], as demonstrated for this structure. From the heralding rate, we then provide a more quantitative estimate of the number of pulses - and thus of the acquisition time - that would be required in an experiment in order to assess the mechanical entanglement.

The nonclassical character of the mechanical quantum state can be indirectly assessed through the optical field. For this, the information stored in the phonon state has to be transferred back to the cavity field. This is achieved thanks to the antiStokes process induced by a second pulse of frequency $\omega_{L}=$ $\omega_{c}+J_{c}-\Omega_{+}$onto the cavity 1 [see $R$ arrow in Fig. 3(c)]. Such an excitation condition is resonant with the beam-splitter interaction [4]

$$
\hat{\mathcal{H}}_{g} \approx \frac{g}{\sqrt{2}}\left(\delta \hat{a}_{+}^{\dagger} \hat{b}_{+}+\delta \hat{a}_{+} \hat{b}_{+}^{\dagger}\right)
$$

which swaps the $\hat{b}_{+}$phonon with an $\hat{a}_{+}$fluctuation. The action of Eq. (6) on the written state produces

$$
\begin{aligned}
\left|\psi_{R}\right\rangle= & c_{00}^{\prime \prime}|0\rangle_{c_{1}} \otimes\left(|10\rangle_{m}+e^{i \varphi^{\prime \prime}}|01\rangle_{m}\right) \otimes|0\rangle_{c_{2}} \\
& +c_{10}^{\prime \prime}|1\rangle_{c_{1}} \otimes\left(1+e^{i \varphi^{\prime \prime}}\right)|00\rangle_{m} \otimes|0\rangle_{c_{2}} \\
& +c_{01}^{\prime \prime}|0\rangle_{c_{1}} \otimes\left(1+e^{i \varphi^{\prime \prime}}\right)|00\rangle_{m} \otimes|1\rangle_{c_{2}}+\cdots .
\end{aligned}
$$

The corresponding cavity-1 intensity involves the second term of Eq. (7) and reads

$$
\mathcal{I}_{1}=\left|c_{10}^{\prime \prime}\right|^{2}\left[1+\cos \left(\varphi^{\prime \prime}\right)\right],
$$

where $\varphi^{\prime \prime}=\varphi_{0}+\Delta \Omega_{m} t^{\prime \prime}$ depends on the delay from the write procedure. Equation (8) reveals a full contrast interference pattern providing means to reveal the entangled nature of the mechanical state. Note that one could equally detect the cavity 2 field and obtain the same result. The expected interferences can be reconstructed by integrating the emission received by a single-photon detector over multiple runs where the delay between the write and readout pulse is gradually increased, as shown in Fig. 3(b) and detailed below.

These simplified and intuitive pictures based on pure states, which were presented above, can be supported by a numerical study of the full system dynamics, which we carried out by using the theoretical tools to model open quantum systems [45]. In this analysis, in order to be able to account for the sizable classical field component induced by the driving laser, we separated the total fields into the sum of their classical and quantum-fluctuation components according to $\hat{a}_{ \pm}=\alpha_{ \pm}+\delta \hat{a}_{ \pm}$and $\hat{b}_{ \pm}=\beta_{ \pm}+\delta \hat{b}_{ \pm}$. The classical evolution is governed by the following set of equations:

$$
\begin{aligned}
i \dot{\alpha}_{ \pm}= & \left( \pm J_{c}+g_{0} \sqrt{2} \operatorname{Re}\left(\beta_{ \pm}\right)-i \frac{\kappa_{ \pm}}{2}\right) \alpha_{ \pm} \\
& +g \sqrt{2} \operatorname{Re}\left(\beta_{\mp}\right) \alpha_{\mp}+\frac{F(t)}{\sqrt{2}} e^{-i\left(\omega_{L}-\omega_{c}\right) t} \\
i \dot{\beta}_{ \pm}=\left(\Omega_{+}\right. & \left.-i \frac{\gamma_{ \pm}}{2}\right) \beta_{ \pm}+\Omega_{-} \beta_{\mp}+\frac{g}{\sqrt{2}}\left(\alpha_{+}^{*} \alpha_{ \pm}+\alpha_{-}^{*} \alpha_{\mp}\right),
\end{aligned}
$$

which are written here in the frame rotating at the cavitymode frequency $\omega_{c}$ and on the basis of the symmetric and antisymmetric modes $\alpha_{ \pm}$and $\beta_{ \pm}$to account for the unbalanced losses $\kappa_{ \pm}$discussed previously. However, we consider equal mechanical losses $\gamma_{ \pm}=2 \pi \times 3.75 \mathrm{kHz}$ which is valid given the large timescales involved. The dynamics of quantum fluctuations is treated via a master equation for the density matrix in the displaced reference frame set by the classical field [46]

$$
\begin{aligned}
\frac{d \hat{\rho}}{d t}= & -i\left[\hat{\mathcal{H}}_{f}, \hat{\rho}\right]+\frac{1}{2} \sum_{j= \pm} \kappa_{j} \hat{\mathcal{D}}\left[\delta \hat{a}_{j}\right] \hat{\rho} \\
& +\frac{1}{2} \sum_{j= \pm}\left[\gamma_{j}\left(\bar{n}_{\mathrm{th}}+1\right) \hat{\mathcal{D}}\left[\delta \hat{b}_{j}\right] \hat{\rho}+\gamma_{j} \bar{n}_{\mathrm{th}} \hat{\mathcal{D}}\left[\delta \hat{b}_{j}^{\dagger}\right] \hat{\rho}\right]
\end{aligned}
$$

where $\bar{n}_{\text {th }}=\left[\exp \left(\Omega_{+} / k_{B} T\right)-1\right]^{-1}$ is the mean thermal phonon occupation and $\hat{\mathcal{D}}[\hat{o}] \hat{\rho}=\hat{o}^{\dagger} \hat{o} \hat{\rho}+\hat{\rho} \hat{o}^{\dagger} \hat{o}-2 \hat{o} \hat{\rho} \hat{o}^{\dagger} \mathrm{de}-$ scribe the dissipations to the environment for each mode. The fluctuation Hamiltonian reads

$$
\begin{aligned}
\hat{\mathcal{H}}_{f}= & \sum_{j=1,2}\left[\Omega_{j} \delta \hat{b}_{j}^{\dagger} \delta \hat{b}_{j}+2 g_{0} \operatorname{Re}\left(\beta_{j}\right) \delta \hat{a}_{j}^{\dagger} \delta \hat{a}_{j}\right] \\
& +\sum_{j=1,2} g_{0}\left[\alpha_{j}^{*} \delta \hat{a}_{j}+\alpha_{j} \delta \hat{a}_{j}^{\dagger}\right]\left[\delta \hat{b}_{j}^{\dagger}+\delta \hat{b}_{j}\right] \\
& +\sum_{j=1,2} g_{0} \delta \hat{a}_{j}^{\dagger} \delta \hat{a}_{j}\left(\delta \hat{b}_{j}^{\dagger}+\delta \hat{b}_{j}\right)+J_{c}\left[\delta \hat{a}_{1}^{\dagger} \delta \hat{a}_{2}+\delta \hat{a}_{2}^{\dagger} \delta \hat{a}_{1}\right],
\end{aligned}
$$

given the inverse transformations $\delta \hat{a}_{1,2}=\left(\delta \hat{a}_{+} \pm \delta \hat{a}_{-}\right) / \sqrt{2}$, $\delta \hat{b}_{1,2}=\left(\delta \hat{b}_{+} \pm \delta \hat{b}_{-}\right) / \sqrt{2}$, and similarly for the classical fields. Note that we kept here the nonlinear optomechanical contributions [third term of Eq. (12)] for an exact description of the system [46].

\section{RESULTS AND DISCUSSION}

The write procedure is conducted with a Gaussian excitation at a time $t_{W}=30 \mathrm{~ns}$, of amplitude $\mathcal{A}_{W}=10^{3} \kappa_{-}$, standard deviation $\sigma_{t}=3.85 \mathrm{~ns}$, and frequency $\omega_{L}=\omega_{c}+J_{c}+\Omega_{+}$on cavity 1 . Note that one could equally excite the antisymmetric mode at $\omega_{L}=\omega_{c}-J+\Omega_{+}$as prescribed by Eq. (2). The driving pulse results in a large classical field amplitude for the cavity mode. This acts as a source for the mechanical field and produces a sizable phonon occupation in both mechanical modes, thanks to the optical coupling between the two cavities. In this way, the normal modes $\hat{a}_{+}$and $\hat{b}_{+}$ 

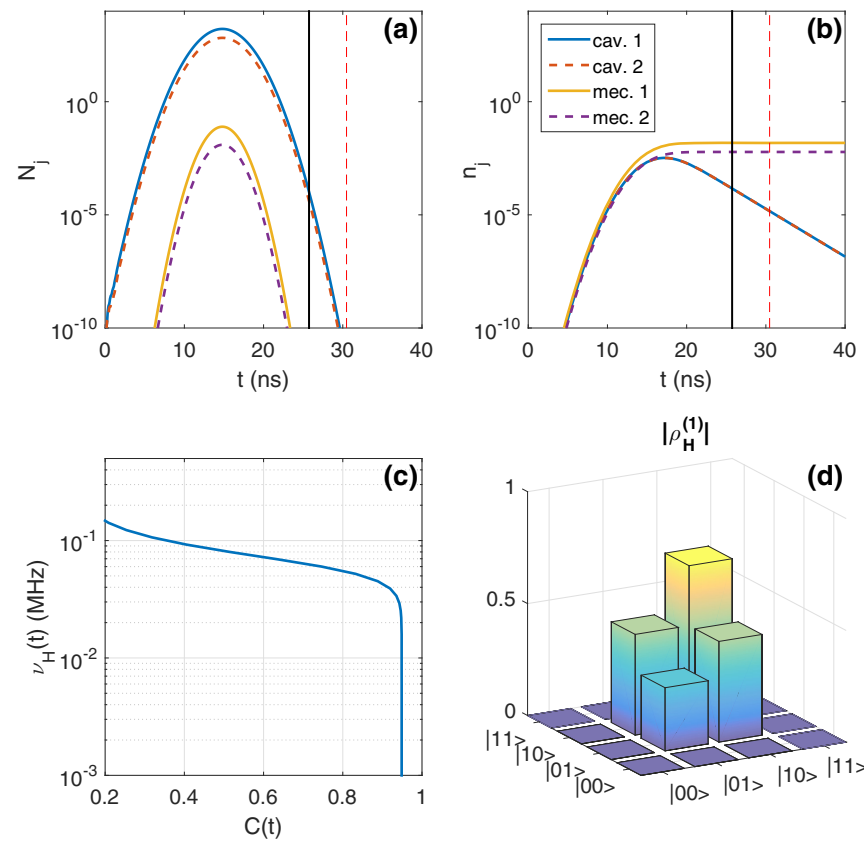

FIG. 4. (Color online) Write procedure: Log scale (a) classical and (b) fluctuation occupations versus time. Blue and dashed-red lines represent cavity modes 1 and 2, yellow and dashed purple lines represent mechanical modes 1 and 2 . The vertical black line marks the time $t_{q}$, starting from which quantum fluctuations become dominant. (c) Parametric plot showing the computed heralding rate $v_{\mathcal{H}}(t)$ versus the concurrence $\mathcal{C}(t)$. (d) Absolute value of the heralded mechanical density matrix $\hat{\rho}_{\mathcal{H}}^{(1)}$ at the $\mathcal{C}_{\max }$ time [vertical red dashed line in panels (a) and (b)] conditioned on the detection of a single photon out of cavity 1 .

are excited without needing to drive the field in the second cavity. Figures 4(a) and 4(b) show, respectively, the classical and quantum fluctuation occupations. We observe that the classical components, which temporarily displace the system to coherent states [47], vanish along with the input pulse. Starting from a time $t_{q} \simeq 25 \mathrm{~ns}$ (vertical black line), quantum fluctuations dominate over the classical components and decay according to the respective lifetimes. The quantity $t_{q}$ defines a time horizon past which the system evolves freely and can display quantum correlations. Importantly, a compromise must be made on the pulse duration, which should be set (i) long enough to accurately excite the sideband without leaking too much on the main cavity frequency and (ii) short enough to allow for a sufficiently large occupation of the quantum fluctuations at time $t_{q}$. Besides, the driving amplitude was set to maximize the heralding success rate past $t_{q}$ while preserving a negligible two-phonon probability.

The heralding at a time $t_{\mathcal{H}}>t_{q}$ is modeled via the projection of the density matrix onto the single-photon subspace $[10,26]$ of cavity 1

$$
\begin{gathered}
\hat{\rho}_{\mathcal{H}}^{(1)}=\frac{\hat{\mathcal{P}}_{1} \hat{\rho}\left(t_{\mathcal{H}}\right) \hat{\mathcal{P}}_{1}^{-1}}{\operatorname{Tr}\left[\hat{\mathcal{P}}_{1} \hat{\rho}\left(t_{\mathcal{H}}\right)\right]}, \\
\hat{\mathcal{P}}_{1}=|1\rangle_{c_{1}}\left\langle\left. 1\right|_{c_{1}} \otimes \hat{\mathbb{I}}_{m_{1}} \otimes \hat{\mathbb{I}}_{m_{2}} \otimes \hat{\mathbb{I}}_{c_{2}} .\right.
\end{gathered}
$$

From $t_{q}$, we consider in Eq. (13) the full density matrix of the system obtained by including the classical contributions as coherent states $\left|\alpha_{ \pm}\right\rangle$and $\left|\beta_{ \pm}\right\rangle$and applying a multimode displacement operator $\hat{\mathcal{D}}\left(\alpha_{ \pm}, \beta_{ \pm}\right)$to $\hat{\rho}$. The heralding efficiencytherefore the degree of mechanical entanglement-is computed through the concurrence $\mathcal{C}$ [48] of the reduced density matrix $\hat{\rho}_{m}=\operatorname{Tr}_{\hat{a}_{1}, \hat{a}_{2}}\left(\hat{\rho}_{\mathcal{H}}\right)$ restrained to the 0 and 1 phonon subspaces. It displays a large maximum value of $\mathcal{C}_{\max }=0.95$ from $t_{\mathcal{H}} \simeq 30 \mathrm{~ns}$ [see vertical dashed lines in Figs. 4(a) and 4(b)]. Corresponding to this value, a mechanical Bell state storing a delocalized phonon is formed, as revealed by the $\left|\hat{\rho}_{\mathcal{H}}^{(1)}\right|$ visual shown in Fig. 4(d). The imbalance in the diagonal elements is a consequence of having driven a single cavity only. We have computed a fidelity $\mathcal{F}=0.97$ to the $(|10\rangle+|01\rangle) / \sqrt{2}$ Bell state. Obviously, the heralding time would be random in an experiment and the detector should target the $t>t_{q}$ time window where $\mathcal{C} \sim \mathcal{C}_{\max }$, while avoiding to monitor earlier times, for the procedure to fully succeed. The entangled state lives as long as the mechanical lifetime $\tau_{m}=2 \pi / \gamma_{1,2}$. Given the very high quality factor characterizing nanobeam cavities, combined to the $\mathrm{GHz}$ range of the frequency of the mechanical modes, one could experimentally reach $\tau_{m}=277 \mathrm{~ms}$ [40] which is promising for quantum repeater technology [27]. When aiming at a concurrence $\mathcal{C}>0.9$, the heralding rate achieved in our simulations was $\nu_{\mathcal{H}}=\kappa_{1,2} n_{1,2} \sim 3 \times 10^{-2} \mathrm{MHz}$, where $\kappa_{1,2}=\left(\kappa_{+}+\kappa_{-}\right) / 2$, as shown in Fig. 4(c). Finally, we point out that, in addition to a large value of the simulated concurrence, in order to assess our target entangled state it is required that the twoand higher- photon terms in the full mechanical density matrix be negligible as it is the case here. This is simply achieved by controlling the amplitude of the driving field. In particular in our simulation, the cavity occupations at $t_{\mathcal{H}}$ amounts to $n_{1,2} \simeq 10^{-4}$ which corresponds to an effective optomechanical coupling $\left\langle\hat{a}_{1,2}\right\rangle g_{0} \simeq 2 \pi \times 86 \mathrm{kHz} \ll \Omega_{1,2}$.

The readout step involves a second input pulse having the same width as the write pulse, and amplitude $\mathcal{A}_{R}=5 \times 10^{3} \kappa_{-}$ delayed by $\Delta T=t_{R}-t_{W}$. The central frequency of the pulse is tuned to the anti-Stokes $\hat{a}_{+}$sideband $\omega_{L}=\omega_{c}+J_{c}-\Omega_{+}$ and aims at transferring the information about the phonon mode back to the optical field. As discussed previously, the nondegeneracy of the mechanical modes $\Delta \Omega=\Omega_{2}-\Omega_{1} \simeq$ $2 \pi \times 50 \mathrm{MHz}$, resulting from the sample imperfections, introduces a relative phase factor $\varphi=\varphi_{0}+\Delta \Omega t$ between the modes which is associated with an interference pattern in the emitted light. The latter is revealed by varying the write and readout pulse delay $\Delta T$ and integrating the corresponding signals [see Fig. 3(b)]. Figures 5(a) and 5(b) show the classical and fluctuations contributions, respectively, to the occupations in a realization where the cavity intensity reaches a maximum [dashed white line in Fig. 5(c)]. Once again, quantum fluctuations dominate after the driving pulse has decayed (vertical black line). Starting from this time, the quantum interference should be recorded. From the Fig. 5(b) inset one can see the $\hat{b}_{+}$mode being emptied by the optical field as revealed in the $\hat{b}_{1}$ and $\hat{b}_{2}$ simultaneous population decay.

Varying gradually the delay $\Delta T$ between the write and readout pulses and specifically $t_{R}$ reveals the predicted interference pattern of period $\mathcal{T}=2 \pi / \Delta \Omega_{m} \simeq 125 \mathrm{~ns}$, as shown in Fig. 5(c). The field in cavity 2 obviously displays a similar behavior (not shown). Note that, to obtain a vertical 

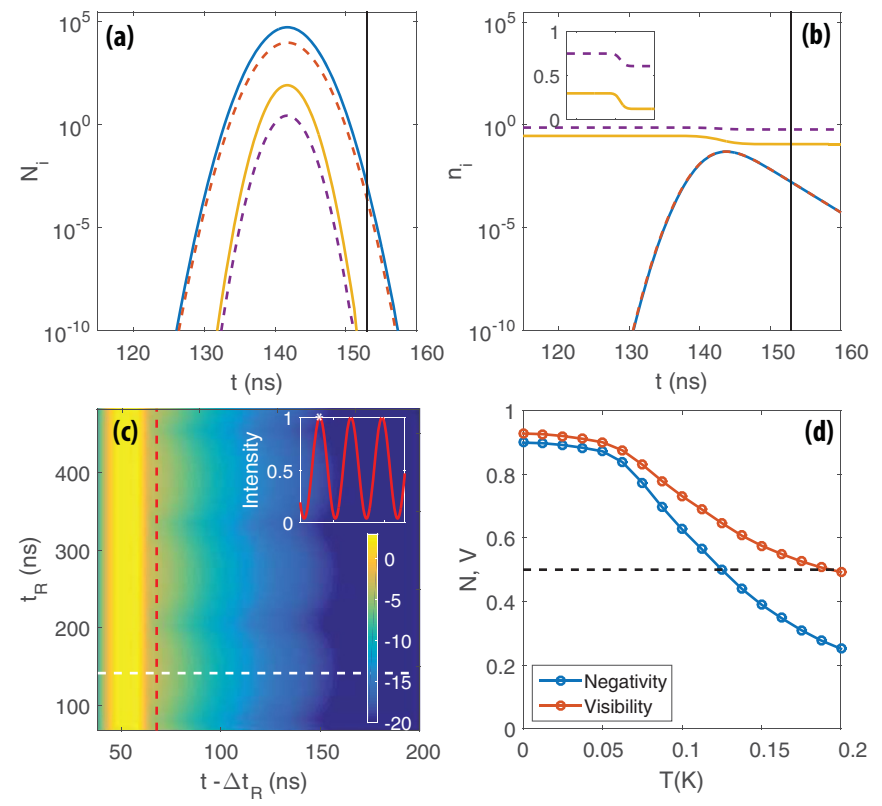

FIG. 5. (Color online) Readout procedure: Logarithmic scale (a) classical and (b) fluctuation contributions to the state occupations versus time, as computed for a given realization of the readout procedure. The inset of panel (b) shows the mechanical occupation in linear scale. (c) The field intensity in cavity 1 , averaged over several runs, as a function of the readout $t_{R}$ and shifted evolution time $t-\Delta t_{R}$ where $\Delta t_{R}=t_{R}^{(j)}-t_{R}^{(1)}$ for run number $j$. The inset shows a normalized cut along the vertical red dashed line, from which the visibility $\mathcal{V}$ can be inferred. The horizontal white dashed line corresponds to the evolutions in panels (a) and (b) where the intensity is at a maximum. (d) Simulated temperature dependence of the negativity and the fringe visibility.

pattern in the plot (instead of an oblique one), the readout pulses have been shifted so that the $x$ axis shows the shifted evolution time $t-\Delta t_{R}$ where $\Delta t_{R}=t_{R}^{(j)}-t_{R}^{(1)}$ and $j$ is the run index. This corresponds to what would be obtained varying the phase $\varphi_{0}$ of the write pulse instead of $\Delta T$. A normalized cut along the vertical axis (dashed-red line), taken at fixed delay from $t_{R}$, is plotted in the inset. It shows a fringe visibility as large as $\mathcal{V} \simeq 0.92$. The interference carries a signature of the relative phase between the two confined phonon modes. This can be related to the amount of phonon entanglement. In particular, a fully separable state would result in a visibility $\mathcal{V}_{c}=0.5$ [26]. This value sets a lower bound, below which the entanglement may still be present in the system, but cannot be directly inferred from the interference pattern. The average cavity occupation at this value of $t_{R}$ amounts to $n_{1}\left(t_{R}\right) \simeq 5 \times 10^{-4}$, which corresponds to a photoemission rate of $v_{R}=0.2 \mathrm{MHz}$. It is interesting at this stage to provide a rough estimate of the experimental requirements to obtain a sufficiently-well-resolved interference pattern. For a Poissonian source, one needs approximately $10^{4}$ pulses in order to expect a $1 \%$ fluctuation of the photon number. Given the present estimate of $v_{R}$ and assuming a delay of $30 \mathrm{~ns}$ between pulses, this would require an acquisition time of about $15 \mathrm{~s}$. This value has to be further corrected in order to account for the single-photon-detector efficiency. State- of-the-art superconducting single-photon detectors nowadays display an efficiency ranging between $10 \%$ and $90 \%$ at telecom wavelengths relevant to this proposal, with $\mathrm{kHz}$-low dark count rates $[49,50]$. These values would result in an acquisition time of roughly one minute. Assuming that one period $\mathcal{T}=125 \mathrm{~ns}$ of the interference pattern of Fig. 5(c) is experimentally resolved with $\sim 100$ time bins, we expect that a few hours of integration and a few million pulses are finally necessary.

At zero temperature we obtained a Bell state with high fidelity, which could be characterized by the concurrence, if restricting the analysis to the subspace with 0 - and 1-phonon states. However, at finite temperature a non-negligible thermal occupation may result in a sizable contribution from states with $n>1$ phonons. In this case, an entanglement measure appropriate to the larger Hilbert space is needed. The concurrence was recently extended to four-dimensional spaces [51]. Its computation, however, is a demanding numerical task for $n \geqslant 4[52,53]$ and is therefore not suitable in the general case we want to consider here. The Bell character of our states naturally suggest to deploy an entanglement monotone derived from positive partial transpose criterion; namely, the negativity $[54,55] \mathcal{N}=\left\|\hat{\rho}_{m}^{\Gamma_{1,2}}\right\|_{1}-1$, where $\hat{\rho}_{m}^{\Gamma_{1,2}}$ is the partial transpose of $\hat{\rho}_{m}$ and $\left\|\hat{\rho}_{m}^{\Gamma_{1,2}}\right\|_{1}$ is its trace norm. The above argument using quantum states can be extended to mixed states in order to estimate a lower bound on the visibility resulting from an entangled state, assuming in particular that the mechanical modes initially lie in the product of two thermal states. We analytically derive the heralded mechanical density matrix at finite temperature in the Appendix $A$ and recover the lower bound of $\mathcal{V}_{c} \simeq 0.5$ to the fringe visibility in the Appendix B.

Besides, the impact of state-of-the-art cooling temperature $T$ [33] on our protocol was computed by direct master equation simulations, and the results are presented in Fig. 5(d), where $\mathcal{N}$ and $\mathcal{V}$ are plotted versus $T$. We see, as expected, a clear correlation between $\mathcal{N}$ and $\mathcal{V}$. They both start decreasing from $T \simeq 0.05 \mathrm{~K}$. Entanglement survives in the temperature range we considered, and the visibility reaches the lower bound $\mathcal{V}_{c}$ at $T_{c} \simeq 0.2 \mathrm{~K}$. Above this temperature, our readout procedure cannot directly assess the presence of entanglement, and a full quantum tomography of the state would be instead required. Finally, the impact of the pure dephasing imposed by other mechanical resonances was analyzed in Ref. [26] and was shown to be negligible in the typical photonic crystal parameter range.

\section{CONCLUSION}

We proposed a realistic photonic crystal architecture based on coupled nanobeams and computed its optomechanical properties. We showed that this structure should allow us to achieve on-chip remote entanglement between the localized acoustic phonon modes, mediated by the optical field, with no need for an additional beam splitter as in typical entanglement schemes $[11,28]$. Our protocol achieves high heralding rates and is still effective at accessible cooling temperature for silicon-based systems. The characteristic long mechanical lifetime makes the present system very promising for demonstrating macroscopic entanglement and for the future of hybrid quantum logic circuits. 


\section{ACKNOWLEDGMENT}

We thank A. Feofanov for discussions on the experimental feasibility. This work was supported by the Swiss National Science Foundation through Project No. 200020_149537.

\section{APPENDIX A: HERALDED DENSITY MATRIX}

In the present protocol, the system is driven around the symmetric cavity mode resonance at $\omega_{+}=\omega_{c}+J_{c}$. Because $2 J \gg \Omega_{1,2}$, the contribution from the antisymmetric mode can be safely disregarded. Under such an assumption we are left with a three-mode system, whose optomechanical interaction reduces to

$$
\hat{\mathcal{H}}_{g} \approx g_{+} \hat{a}_{+}^{\dagger} \hat{a}_{+}\left(\hat{b}_{+}^{\dagger}+\hat{b}_{+}\right) .
$$

Here, $\quad \hat{b}_{+}=\left(\hat{b}_{1}+\hat{b}_{2}\right) / \sqrt{2}$ and $g_{+}=g_{0} / \sqrt{2}$. At low temperature, the cavity mode can be assumed to lie in its vacuum state, described by the density matrix $\hat{\rho}_{c}^{0}=|0\rangle\langle 0|$. The mechanical modes on the other hand are characterized by resonant frequencies in the $\mathrm{GHz}$ range, and states with a finite thermal phonon occupation must be assumed. Then the density matrices of the mechanical modes 1 and 2 are expressed as

$$
\hat{\rho}_{m_{1,2}}^{0}=(1-p) \sum_{n=0}^{N-1} p^{n}|n\rangle\langle n|=(1-p) \sum_{n=0}^{N-1} p^{n} \hat{\rho}_{n},
$$

where $N$ is the maximal occupation number assumed when truncating the Hilbert space and $p=\bar{n}_{\mathrm{th}} /\left(1+\bar{n}_{\mathrm{th}}\right)$. The number $N$ in our calculations is increased until convergence is reached. However, we discuss more specifically the case $N=$ 2 hereafter, because it leads to compact analytical expressions for the density operators and allows for a simple analytical expression of the concurrence parameter. We assume that the initial density matrix is separable, i.e., $\hat{\rho}_{0}=\hat{\rho}_{c}^{0} \otimes \hat{\rho}_{m}^{0}$ where

$$
\hat{\rho}_{m}^{0}=\hat{\rho}_{m_{1}}^{0} \otimes \hat{\rho}_{m_{2}}^{0}=(1-p)^{2} \sum_{n, m=0}^{N-1} p^{(n+m)} \hat{\rho}_{n, m} .
$$

Here we have used the notation $\hat{\rho}_{n, m}=|n, m\rangle\langle n, m|$. The Stokes process used for the write procedure resonantly

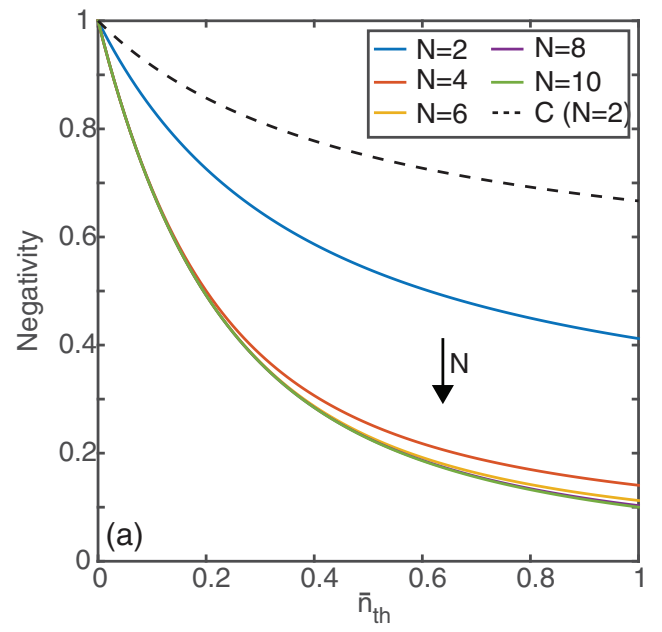

generates two-mode squeezing through the corresponding linearized term (3). Under this interaction the system density matrix evolves according to $\hat{\rho}(t)=\hat{\mathcal{U}} \hat{\rho}_{0} \hat{\mathcal{U}}^{\dagger}$ where $\hat{\mathcal{U}}=\exp \left(-i \hat{\mathcal{H}}_{g} t\right)$. The latter is roughly approximated to $\hat{\mathcal{U}} \simeq \hat{\mathbb{I}}-i \hat{\mathcal{H}}_{g} \Delta t+O\left(\Delta t^{2}\right)$ for a short pulse duration $\Delta t$. Therefore, one has $\hat{\rho}(\Delta t) \simeq\left(1-i \hat{\mathcal{H}}_{g} \Delta t\right) \hat{\rho}_{0}\left(1+i \hat{\mathcal{H}}_{g}^{\dagger} \Delta t\right)$. The heralding procedure, which projects $\hat{\rho}(t)$ onto its one-photon subspace, only retains the action of the term proportional to $\hat{\mathcal{H}}_{g} \hat{\rho}_{0} \hat{\mathcal{H}}_{g}^{\dagger}$ after the write procedure. By tracing this state over the cavity mode, the heralded density matrix of the mechanical system is then obtained:

$$
\begin{aligned}
\hat{\rho}_{m}^{(H)}= & (1-p)^{2} \sum_{n, m=0}^{N-1} p^{(n+m)}\left[(n+1) \hat{\rho}_{n+1, m}+(m+1) \hat{\rho}_{n, m+1}\right. \\
& \left.+\sqrt{(n+1)(m+1)}\left(e^{+i \varphi} \hat{\rho}_{n+1, m}^{n, m+1}+e^{-i \varphi} \hat{\rho}_{n, m+1}^{n+1, m}\right)\right],
\end{aligned}
$$

where we use $\hat{\rho}_{i, j}^{k, l}=|i, j\rangle\langle k, l|$ for the off-diagonal matrix terms. The relative phase factors are introduced by the action of $\hat{b}_{2}^{\dagger}$ in the frame rotating at the frequency $\Omega_{2}$ where $\varphi=\Delta \Omega t$. In the simple case where $N=2$ (i.e., assuming no more than one phonon per mode, valid for very low temperature where $p \ll 1$ ), we obtain

$$
\hat{\rho}_{m}^{(H)}=\frac{1}{2(p+1)}\left[\begin{array}{c}
\hat{\rho}_{1,0}+\hat{\rho}_{0,1}+2 p \hat{\rho}_{1,1} \\
+e^{+i \varphi} \hat{\rho}_{1,0}^{0,1}+e^{-i \varphi} \hat{\rho}_{0,1}^{1,0}
\end{array}\right]
$$

upon trace normalization. At zero temperature where $p=0$, Eq. (A5) reduces to

$$
\hat{\rho}_{m}^{(H)}=\frac{1}{2}\left(\hat{\rho}_{1,0}+\hat{\rho}_{0,1}+e^{+i \varphi} \hat{\rho}_{1,0}^{0,1}+e^{-i \varphi} \hat{\rho}_{0,1}^{1,0}\right) ;
$$

namely, the density matrix associated with the pure Bell state $|\psi\rangle=\left(|10\rangle+e^{i \varphi}|01\rangle\right) / \sqrt{2}$. The concurrence of the $4 \times 4$ mixed state (A5) straightforwardly reads

$$
\mathcal{C}=\frac{1}{1+p}=1-\frac{\bar{n}_{\mathrm{th}}}{2 \bar{n}_{\mathrm{th}}+1}
$$

and is plotted in Fig. 6(a) (dashed black line).

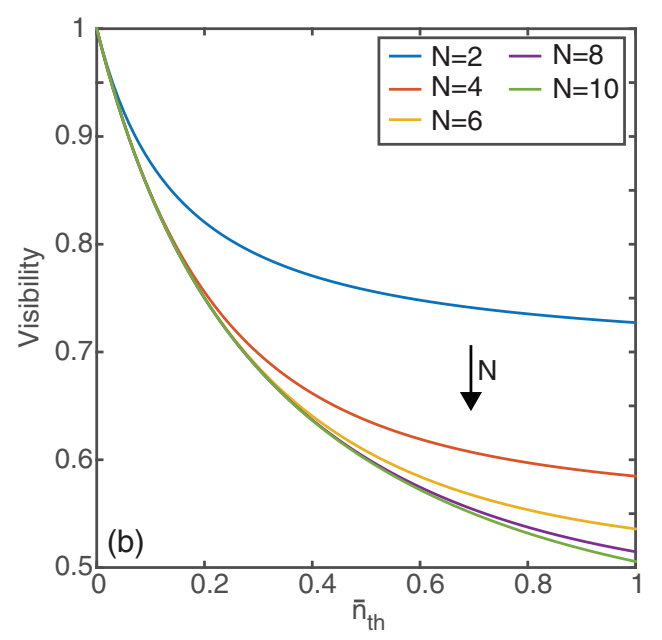

FIG. 6. (Color online) (a) Negativity $\mathcal{N}$ versus $\bar{n}_{\text {th }}$ for several values of $N$. The black dashed line shows the concurrence $\mathcal{C}$ in the case $N=2$. (b) Corresponding visibility computed from Eq. (B3). The arrows point the direction of increasing values of $N$. 
For $N \geqslant 2$, the degree of entanglement can be evaluated through the negativity $\mathcal{N}=\sum_{i}\left|\lambda_{i}\right|-\lambda_{i}$ where $\lambda_{i}$ are the negative eigenvalues of the partial transpose of $\hat{\rho}_{m}^{(H)}$ with respect to one of the mechanical modes. One easily finds, e.g., for $N=2$, that only one eigenvalue $\lambda=[p-$ $\left.\left(1+p^{2}\right)^{1 / 2}\right] / 2(1+p)$ is negative independently of $p$, and therefore

$$
\mathcal{N}=\frac{\sqrt{1+p^{2}}-p}{1+p}=\left(\sqrt{1+p^{2}}-p\right) \mathcal{C} .
$$

The cases where $N>2$ are presented in Fig. 6(a) (see legend).

\section{APPENDIX B: READOUT INTENSITY}

The anti-Stokes process used for the readout procedure drives the process associated with the interaction (6). When applying this operator onto the heralded density matrix Eq. (A4) and retaining as in the write case only terms with one photon, the resulting density matrix reads

$$
\begin{aligned}
\hat{\rho}_{R}= & (1-p)^{2} \sum_{n, m=0}^{N-1} p^{(n+m)}\left[(n+1)^{2}+(m+1)^{2}\right. \\
& +2(n+1)(m+1) \cos \varphi] \hat{\rho}_{n, m}
\end{aligned}
$$

$$
\begin{aligned}
& +(1-p)^{2} \sum_{n, m=0}^{N-1} p^{(n+m)}\left[n(m+1) \hat{\rho}_{n-1, m+1}\right. \\
& \left.+m(n+1) \hat{\rho}_{n+1, m-1}\right]+\cdots,
\end{aligned}
$$

where we indicate only the diagonal terms explicitly. The field intensity of the cavity mode, associated with Eq. (B1) is given by

$$
\begin{aligned}
\mathcal{I}(\varphi)= & (1-p)^{2} \sum_{n, m=0}^{N-1} p^{(n+m)}[(n+m+1)(n+m+2) \\
& +2(n+1)(m+1) \cos \varphi] .
\end{aligned}
$$

From there one can deduce the fringes visibility as $\mathcal{V}=$ $\left(\mathcal{I}_{\max }-\mathcal{I}_{\text {min }}\right) /\left(\mathcal{I}_{\text {max }}+\mathcal{I}_{\text {min }}\right)$, where $\mathcal{I}_{\max }=\mathcal{I}(0)$ and $\mathcal{I}_{\text {min }}=$ $\mathcal{I}(\pi)$, to obtain

$$
\mathcal{V}=\frac{\sum_{n, m=0}^{N-1} 2 p^{(n+m)}(n+1)(m+1)}{\sum_{n, m=0}^{N-1} p^{(n+m)}(n+m+1)(n+m+2)}
$$

We show in Fig. 6(b) a plot of $\mathcal{V}$ versus $\bar{n}_{\text {th }}$ for several values of $N$ (see legend). Within the range of values considered for $\bar{n}_{\text {th }}$, convergence is achieved for $N \geqslant 8$.
[1] T. J. Kippenberg and K. J. Vahala, Science 321, 1172 (2008).

[2] I. Favero and K. Karrai, Nat. Photonics 3, 201 (2009).

[3] F. Marquardt and S. M. Girvin, Physics 2, 40 (2009).

[4] M. Aspelmeyer, T. J. Kippenberg, and F. Marquardt, Rev. Mod. Phys. 86, 1391 (2014).

[5] T. Corbitt, Y. Chen, F. Khalili, D. Ottaway, S. Vyatchanin, S. Whitcomb, and N. Mavalvala, Phys. Rev. A 73, 023801 (2006).

[6] T. P. Purdy, P.-L. Yu, R. W. Peterson, N. S. Kampel, and C. A. Regal, Phys. Rev. X 3, 031012 (2013).

[7] V. Peano, H. G. L. Schwefel, C. Marquardt, and F. Marquardt, arXiv:1502.06423.

[8] R. Almog, S. Zaitsev, O. Shtempluck, and E. Buks, Phys. Rev. Lett. 98, 078103 (2007).

[9] M. R. Vanner, M. Aspelmeyer, and M. S. Kim, Phys. Rev. Lett. 110, 010504 (2013).

[10] C. Galland, N. Sangouard, N. Piro, N. Gisin, and T. J. Kippenberg, Phys. Rev. Lett. 112, 143602 (2014).

[11] S. Pirandola, D. Vitali, P. Tombesi, and S. Lloyd, Phys. Rev. Lett. 97, 150403 (2006).

[12] D. Vitali, S. Gigan, A. Ferreira, H. R. Böhm, P. Tombesi, A. Guerreiro, V. Vedral, A. Zeilinger, and M. Aspelmeyer, Phys. Rev. Lett. 98, 030405 (2007).

[13] L. Zhou, Y. Han, J. Jing, and W. Zhang, Phys. Rev. A 83, 052117 (2011).

[14] U. Akram, W. Munro, K. Nemoto, and G. J. Milburn, Phys. Rev. A 86, 042306 (2012).

[15] R. Ghobadi, S. Kumar, B. Pepper, D. Bouwmeester, A. I. Lvovsky, and C. Simon, Phys. Rev. Lett. 112, 080503 (2014).

[16] M. Pinard, A. Dantan, D. Vitali, O. Arcizet, T. Briant, and A. Heidmann, Europhys. Lett. 72, 747 (2005).

[17] M. J. Hartmann and M. B. Plenio, Phys. Rev. Lett. 101, 200503 (2008).
[18] M. Ludwig, K. Hammerer, and F. Marquardt, Phys. Rev. A 82, 012333 (2010).

[19] K. Borkje, A. Nunnenkamp, and S. M. Girvin, Phys. Rev. Lett. 107, 123601 (2011).

[20] W. Ge, M. Al-Amri, H. Nha, and M. S. Zubairy, Phys. Rev. A 88, 022338 (2013).

[21] U. Akram, W. P. Bowen, and G. J. Milburn, New J. Phys. 15, 093007 (2013).

[22] H. Tan, G. Li, and P. Meystre, Phys. Rev. A 87, 033829 (2013).

[23] X.-W. Xu, Y.-J. Zhao, and Y.-x. Liu, Phys. Rev. A 88, 022325 (2013).

[24] J.-Q. Liao, Q.-Q. Wu, and F. Nori, Phys. Rev. A 89, 014302 (2014).

[25] A. Szorkovszky, A. A. Clerk, A. C. Doherty, and W. P. Bowen, New J. Phys. 16, 063043 (2014).

[26] H. Flayac and V. Savona, Phys. Rev. Lett. 113, 143603 (2014).

[27] A. I. Lvovsky, B. C. Sanders, and W. Tittel, Nat. Photonics 3, 706 (2009).

[28] K. C. Lee, M. R. Sprague, B. J. Sussman, J. Nunn, N. K. Langford, X.-M. Jin, T. Champion, P. Michelberger, K. F. Reim, D. England, D. Jaksch, and I. A. Walmsley, Science 334, 1253 (2011).

[29] L.-M. Duan, M. D. Lukin, J. I. Cirac, and P. Zoller, Nature (London) 414, 413 (2001).

[30] A. D. O'Connell, M. Hofheinz, M. Ansmann, R. C. Bialczak, M. Lenander, E. Lucero, M. Neeley, D. Sank, H. Wang, M. Weides, J. Wenner, J. M. Martinis, and A. N. Cleland, Nature (London) 464, 697 (2010).

[31] J. Chan, T. P. M. Alegre, A. H. Safavi-Naeini, J. T. Hill, A. Krause, S. Groblacher, M. Aspelmeyer, and O. Painter, Nature (London) 478, 89 (2011). 
[32] R. Riviere, O. Arcizet, A. Schliesser, and T. J. Kippenberg, Rev. Sci. Instrum. 84, 043108 (2013).

[33] S. M. Meenehan, J. D. Cohen, S. Gröblacher, J. T. Hill, A. H. Safavi-Naeini, M. Aspelmeyer, and O. Painter, Phys. Rev. A 90, 011803(R) (2014).

[34] A. H. Safavi-Naeini, J. T. Hill, S. Meenehan, J. Chan, S. Gröblacher, and O. Painter, Phys. Rev. Lett. 112, 153603 (2014).

[35] E. Gavartin, R. Braive, I. Sagnes, O. Arcizet, A. Beveratos, T. J. Kippenberg, and I. Robert-Philip, Phys. Rev. Lett. 106, 203902 (2011).

[36] K. Makles, T. Antoni, A. G. Kuhn, S. Delglise, T. Briant, P.-F. Cohadon, R. Braive, G. Beaudoin, L. Pinard, C. Michel, V. Dolique, R. Flaminio, G. Cagnoli, I. Robert-Philip, and A. Heidmann, Opt. Lett. 40, 174 (2015).

[37] P. Rath, S. Khasminskaya, C. Nebel, C. Wild, and W. H. P. Pernice, Nat. Commun. 4, 1690 (2013).

[38] B. Khanaliloo, H. Jayakumar, A. C. Hryciw, D. P. Lake, H. Kaviani, and P. E. Barclay, arXiv:1502.01788.

[39] R. Horodecki, P. Horodecki, M. Horodecki, and K. Horodecki, Rev. Mod. Phys. 81, 865 (2009).

[40] J. Chan, A. H. Safavi-Naeini, J. T. Hill, S. Meenehan, and O. Painter, Appl. Phys. Lett. 101, 081115 (2012).

[41] Y. Sato, Y. Tanaka, J. Upham, Y. Takahashi, T. Asano, and S. Noda, Nat. Photonics 6, 56 (2012).

[42] Lumerical Solutions, Inc., http://www.lumerical.com/tcadproducts/fdtd/.
[43] P. Barclay, K. Srinivasan, and O. Painter, Opt. Express 13, 801 (2005)

[44] Comsol Multophysics inc., http://www.comsol.com/comsolmultiphysics.

[45] C. Gardiner and P. Zoller, Quantum Noise: A Handbook of Markovian and Non-Markovian Quantum Stochastic Methods with Applications to Quantum Optics, Springer Series in Synergetics (Springer, New York, 2004).

[46] A. Nunnenkamp, K. Børkje, and S. M. Girvin, Phys. Rev. Lett. 107, 063602 (2011).

[47] N. Bruno, A. Martin, P. Sekatski, N. Sangouard, R. T. Thew, and N. Gisin, Nat. Phys. 9, 545 (2013).

[48] W. K. Wootters, Phys. Rev. Lett. 80, 2245 (1998).

[49] M. G. Tanner, C. M. Natarajan, V. K. Pottapenjara, J. A. O'Connor, R. J. Warburton, R. H. Hadfield, B. Baek, S. Nam, S. N. Dorenbos, E. B. Urea, T. Zijlstra, T. M. Klapwijk, and V. Zwiller, Appl. Phys. Lett. 96, 221109 (2010).

[50] F. Marsili, V. B. Verma, J. A. Stern, S. Harrington, A. E. Lita, T. Gerrits, I. Vayshenker, B. Baek, M. D. Shaw, R. P. Mirin, and S. W. Nam, Nat. Photonics 7, 210 (2013).

[51] K. Audenaert, F. Verstraete, and B. De Moor, Phys. Rev. A 64, 052304 (2001).

[52] P. Rungta, V. Bužek, C. M. Caves, M. Hillery, and G. J. Milburn, Phys. Rev. A 64, 042315 (2001).

[53] P. Rungta and C. M. Caves, Phys. Rev. A 67, 012307 (2003).

[54] G. Vidal and R. F. Werner, Phys. Rev. A 65, 032314 (2002).

[55] M. B. Plenio, Phys. Rev. Lett. 95, 090503 (2005). 